\title{
Management of Dravet syndrome and emerging therapy options
}

Christine Prager J Helen Cross

UCL-Great Ormond Street Institute of Child Health, London, WC1N 1EH

\author{
Address for correspondence \\ Professor JH Cross \\ Clinical Neurosciences \\ UCL-Great Ormond Street Institute of Child Health \\ Guilford Street \\ London WC1N 1EH \\ +442079052981 \\ Email: h.cross@ucl.ac.uk
}




\section{Introduction}

Dravet syndrome (severe myoclonic epilepsy of infancy; MIM 607208) is an epileptic and genetic encephalopathy that is characterised by onset in the first year of life, with prolonged generalized or hemi-clonic seizures often associated with fever, followed by the development of multiple seizure types generally resistant to anti-epileptic drugs, with slowing of neurodevelopment [1]. Long term prognosis for both seizure control and neurodevelopmental outcome is poor [2]. In an online survey of 274 patients with Dravet syndrome, $45 \%$ reported an average of more than 4 tonic-clonic seizures per month, despite being treated with a combination of an average of 3 drugs [3]. This aside, management can be optimised by utilising appropriate medications shown to be of benefit, and avoiding those medications that we know may aggravate seizures. This review focuses on the current drug treatment available for patient with Dravet syndrome as well as agents in development.

\subsection{Clinical description}

. Seizures typically start between 4 and 9 months of age in a normal infant. The first seizure is usually clonic, generalized, or unilateral. It is usually accompanied by fever or mild hyperthermia, or can occur during a bath, or during a viral infection, but can also occur without fever and is often prolonged. Seizures following vaccinations are reported in about $27 \%$ of patients with Dravet syndrome [4], a Dutch study reported that $2.5 \%$ of all seizures after vaccination in the first year of life occur in children with Dravet syndrome [5]. Vaccination may trigger earlier onset of Dravet syndrome in children who, because of an SCN1A mutation, are destined to develop the disease. However, vaccination should not be withheld from children with SCN1A mutations because there is no evidence that vaccinations before or after disease onset affect outcome [6] [7].

The median age of a first status epilepticus has been found to be 8 months of age [8].

Afebrile seizures are quickly associated with febrile seizures and other seizure types develop: 
1 Convulsive seizures, apparently generalized or unilateral, are present throughout the evolution in all patients. Unilateral seizures are the most characteristic. In the youngest patients, they correspond to hemiclonic seizures and often evolve to status epilepticus.

2 Myoclonic seizures appear between the ages 1 and 5 years in approximately $85 \%$ of patients. Their intensity is variable. They can be massive, involving all muscles, particularly axial, or sometimes barely discernible, involving only the axial muscles (head and trunk), with an atonic component, giving a small movement forward or backward, more or less saccadic, described as a "head nodding." They are either isolated or grouped in brief bursts (1-3 s) and are very frequent, occurring several times a day, sometimes incessantly.

3 Atypical absences may present to the age of 12 years, they have a frequent myoclonic component and sometimes an atonic component with a head drop. Peculiar obtundation status is observed in $40 \%$ of patients with impaired consciousness, accompanied by myoclonia or atonic drops with intermixed short periods of compete loss of conscionsness. These periods are generally long-lasting; they appear preferentially in the morning at awakening and are characterized by obtundation with poor responsivity and erratic myoclonus, involving mainly the fingers and orobuccal muscles with drooling, and intercalated by isolated and randomly recurrent massive jerks. When persistent in the evening they can interfere with falling asleep. Convulsive seizures can initiate, occur during, or terminate this type of status. [9] [10]

4 Focal seizures consist of motor seizures or seizures with more complex semiology, including prominent autonomic symptoms. They occur in $43-78.6 \%$ of patients.

5 Tonic seizures are unusual in this syndrome. They resemble the axial tonic seizures of the Lennox Gastaut syndrome [10].

Photo and pattern sensitivity are frequent, they are variable during the course of the disease. Many other stimuli may trigger seizures: hot baths, physical exercise, noisy environments, emotions, and other individual stimuli [11].

\subsection{Epidemiology}


In a retrospective, population-based cohort of all infants born at Kaiser Permanente Northern California during 2007-2010, patients were electronically identified who received $\geq 2$ seizure diagnoses before age 12 months and who were also prescribed anticonvulsants at 24 months. A child neurologist reviewed records to identify infants who met 4 of 5 criteria for clinical Dravet syndrome:

1. normal development before seizure onset

2. $\geq 2$ seizures before age 12 months

3. myoclonic, hemiclonic, or generalized tonic-clonic seizures

4. $\geq 2$ seizures lasting $>10$ minutes

5. refractory seizures after age 2 years.

When these criteria were applied an incidence of 1 per 15700 was determined [12]. SCN1A gene sequencing was performed, $75 \%$ had a de novo SCN1A mutation that was likely to be pathogenic. This incidence was slightly higher than previously reported in a UK study (at least 1: 40900) [13]. A Danish population based study revealed an incidence of 1: 22000 [14].

\subsection{Genetics}

$70-80 \%$ of children with a Dravet phenotype have a mutation of the SCN1A gene, encoding the a1 subunit of the neuronal voltage-gated sodium channel SCN1A (protein name: Nav1.1) [15]. More than 650 SCN1A variants have been discovered in patients with Dravet syndrome, and several others in other epilepsy syndromes [16]. More than half of these are truncation mutations, the others missense. $90 \%$ are de novo, the remaining $10 \%$ of identified mutations are inherited and family members often have milder GEFS+ phenotypes [17]. The nature of the mutation does not allow the clinician to confidently predict the phenotype [18]. PCDH19 mutational analysis is a second-tier test for girls with a Dravet-like picture who do not have SCN1A mutations [19], although the clinical picture is somewhat different, girls having a slightly later age of onset and less a less severe phenotype. Mutations in the SCN2A gene, which codes for the a2subunit of the neuronal sodium channel Nav1.2 also lead to severe epileptic encephalopathies resembling Dravet syndrome [20]. 
1.4. Development, comorbidities and health related quality of life Most patients show a normal development in the first year of life, however at the end of the second year, $60 \%$ show a mild, moderate or severe learning disability. By 15 years of age, over $80 \%$ of affected children have a severe or profound learning disability [13]. There is a dissociated cognitive profile, particularly for patients with SCN1A mutations: speech develops better than visuo-motor function from the first year of life and before cognitive slowing is almost constant from the age of three. There is some evidence for an onset of a visual disorder ('dorsal stream vulnerability') emerging before cognitive decline and even before the full epileptic manifestations, which would support a genetic role in developmental impairment in patients with Dravet syndrome [21].

For patients over the age of three, developmental quotient has been reported as significantly lower for patients exhibiting myoclonic or focal seizures [22]. Otherwise, whether the severity of cognitive impairment is linked to the frequency of seizures, especially convulsive seizures, is disputed [23]. There are series that have failed to detect any significant correlation between cognitive scores and epilepsy variables, i.e. number and age of onset of status epilepticus, age and duration of first seizure, types of seizures during the course, photosensitivity and treatment [22] [24]. Neurological deterioration continues throughout life in all patients into adulthood, with further impairment of speech, mobility and ability for daily activities and dysphagia emerging as late features [25]. Acquired autistic features (33\% of children), behaviour problems (46\%) and acquired motor disorder (36\%) all increase significantly with age. Up to two-thirds of children have inattention/hyperactivity symptoms, one third conduct problems, and three fourths problems with peer relationships. The high prevalence of behavioural problems in patients with Dravet syndrome has significant impact on quality of life independent of epilepsyrelated problems [13]. 
Approximately $15 \%$ of children die before reaching adulthood, most commonly due to SUDEP, complications of status epilepticus or accidental drowning [26].

\subsection{Electroencephalographic features}

There are no specific interictal EEG features. The EEG is often normal at the onset of the illness, followed by a progressive increase in paroxysmal abnormalities. The characteristics of background activity fluctuate, depending on the number and severity of seizures. During wakefulness, background activity remains normal in up to $62 \%$ of patients or shows a slight slowing. In the remaining cases, background activity becomes slow and poorly organized [27]. Two peculiar types of convulsive seizures are considered typical in EEG in Dravet syndrome: the "falsely generalized seizures" and the "unstable seizures". The "falsely generalized" seizures are characterized by a complicated semiology with some degree of discrepancy between clinical and EEG phenomena. The EEG discharges are initially bilateral but become and remain asymmetric during the seizure. The "unstable seizures" are characterized by the topographic changes of the ictal EEG discharge in the same seizure. But the ways of propagation are variable from one seizure to another in the same patient, even in the same recording. The relationship between the clinical manifestations and the accompanying EEG is not always clear [28].

In obtundation status the EEG is usually characterized by one diffuse dysrhythmia of slow waves, intermixed with focal and diffuse spikes sharp waves, and SWs, of higher voltage in the anterior regions and the vertex, without time correspondence between the spikes and the myoclonic jerks except during the intercalated myoclonic fits. Strong sensory stimulation can transiently interrupt the status [28].

Absence seizures in Dravet syndrome are characterized by early- age of onset, a high incidence of irregular and disorganized $3 \mathrm{~Hz}$ GSW morphology, and the frequent association with generalized myoclonic movements [29]. 
Myoclonic manifestations in DS have been reported to be heterogeneous and include nonepileptic myoclonus. The myoclonic seizures can be isolated or grouped in brief bursts of two or three jerks. They predominate in the arms, the axial muscles and the neck. The EEG recording shows generalized SWs or multiple SWs, at $3 \mathrm{~Hz}$ or more, with higher voltage on the frontocentral areas and on the vertex [28].

\subsection{Neuroimaging}

Several studies reporting magnetic resonance imaging (MRI) abnormalities in patients with Dravet syndrome have provided contradicting data regarding occurrence of cerebral and cerebellar atrophy and percentage with hippocampal sclerosis. Hippocampal sclerosis was reported in $10(71 \%)$ in a series of 14 patients [30], but only one of 58 patients in a further series, whereas other abnormalities such as atrophy were found in 12 patients (21\%) [31].

In another study of 30 patients the first MRI was performed at a median age of 1.1 (range $0.2-$ 13.0) years and was normal in 26 patients (87\%). Abnormalities were found in four children: one cortical atrophy at 3.1 years and three temporal lobe abnormalities. Nineteen patients with initially normal MRI had at least one subsequent MRI available for reanalysis; five of them showed new temporal lobe abnormalities, two had developed cortical or cerebellar atrophy at 2.2-2.4 years, and another two had acute changes associated with SE. The frequency of temporal lobe abnormalities was 7 (39\%) of 18 in patients imaged after the age of 3 years, either hippocampal sclerosis or loss of grey-white matter definition in the anterior temporal lobe was observed [32]. However, epidemiology does not support a causative role for SCN1A mutations in malformations of cortical development. The few studies that have addressed the electroclinical characteristics of focal seizures in patients with SCN1A mutations in relation to MRI-visible structural abnormalities could not identify any consistent correlation [33] and surgery in such cases has not been helpful.

In summary, Dravet syndrome is an early onset encephalopathy with specific clinical features and seizure types. Whereas interictal EEG and neuroimaging are non-specific; the ictal EEG 
often reveals specific elements. The encephalopathy seems to a consequence of epilepsy, but the underlying genetic mutation seems to play an additional direct role.

2. Management of seizures

\subsection{General Management}

Management of children with Dravet syndrome does not only involve consideration of drug management of seizures. It is clear that optimal management should include avoidance of seizure triggers, such as photic triggers in those who remain photosensitive, and minimising fever with use of antipyretics for fever, minimizing hot baths, and avoidance of overheating on hot days. Further prolonged seizures warrant prompt treatment, with utilisation of home rescue medication Every patient should have a personalised plan for seizures lasting more than 5 minutes, and indeed in many immediate treatment of any convulsive seizure is warranted in view to the risk of such becoming prolonged. Ultimately there should also be avoidance of antiepileptic drugs that may exacerbate seizures. Exacerbation is well documented for carbamazepine and lamotrigine, also for phenytoin, pregabalin, vigabatrin and gabapentin.

\subsection{Current antiepileptic drugs}

\subsubsection{Valproic acid}

Valproate is a broad spectrum antiepileptic drug, and consequently its greatest value is in the management of generalised epilepsies associated with multiple seizure types [34]. It is considered to be first line treatment for patients with Dravet syndrome, currently the most common antiepileptic drug used in such patients in Europe (85\%). Efficacy for tonic clonic seizures patients with Dravet syndrome is reported between $41 \%$ to $52 \%$ [35] [36] [37].

The European Medicines Agency has issued a warning with regard to use of Valproate in any girl with epilepsy in view of the risk of major congenital malformations as well as cognitive and behavioural impairment in prenatally exposed children [38]. The International League Against Epilepsy, however, in association with the European Academy of Neurology, have highlighted guidelines on consideration of risk-benefit of its use [39]. 
If there are clinical features suggestive of possible mitochondrial disease, mitochondrial genetic testing (specifically mutation of the POLG gene) should be obtained prior to initiation of valproate, as these patients are at substantial risk of valproate-induced, fulminant hepatic failure. Other important adverse effects include pancreatitis, hyperammonemia and blood dyscrasias. Thrombocytopenia is common. Other common side effects include nausea and vomiting, weight gain, sedation, and hair loss [35]. This aside, on a diagnosis or suspicion of Dravet syndrome, Valproate would be the first line drug of choice.

\subsubsection{Clobazam}

Clobazam is a 1,5-benzodiazepine approved as adjunctive treatment of epilepsy in more than 100 countries since its introduction in 1974 [40]. It is considered to be a second-line treatment in Dravet syndrome [41].

In a prospective randomized placebo controlled trial including 235 children in 1998 clobazam was found to be equally effective as carbamazepine and phenytoin for treatment of focal seizure with and without secondary generalization, and primary generalized tonic-clonic seizures without associated absence or myoclonus [42].

Several studies have since examined clobazam use in paediatric populations, with seizure freedom occurring in $9 \%$ to $60 \%$ of patients and responder rates ( $>50 \%$ seizure reduction) ranging from $32 \%$ to $75 \%[43,44]$ even for patient with intractable epilepsy. There is no difference between various aetiologies [45].

Tolerance to treatment is a significant concern for benzodiazepines used as long-term therapy. Several studies examined clobazam tolerance in patients with initial favourable response and found a $50 \%$ rate at a mean follow up of 17 months [46] or 15 months [43].

Main adverse effects are sedation, ataxia, increased salivation and behavioural disinhibition.

\subsubsection{Stirpentol}


Stiripentol was the first antiepileptic drug that obtained an orphan designation at the European Medical Agency (EMA) in 2001 for a specific disease, namely Dravet syndrome. Stiripentol was registered in Europe as an orphan drug in January 2007 for generalised tonic clonic seizures as part of Dravet patients as adjunctive therapy with VPA and CLB, based on the two small confirmatory placebo-controlled trials conducted in France and Italy. 15 (71\%) patients were responders to stiripentol (including nine free of clonic or tonic-clonic seizures), whereas there was only one (5\%) on placebo [47]. The data from the second trial were included in the metaanalysis.

Stiripentol has been shown to inhibit the metabolism of clobazam, especially norclobazam (NCLB) hydroxylation by CYP2C19 [48]. The efficacy of stiripentol however could be related not only to its ability to increase the effective concentration of other drugs, but also to positive modulation of c-aminobutyric acid (GABA)ergic inhibitory neurotransmission [49].

Data from Japan have confirmed responder rates of $61 \%$ and $48 \%$, respectively, for convulsive Dravet seizures in the short and longer term in 23 patients reviewed, despite the fact that about half these patients did not receive valproate and clobazam as comedications. Moreover, duration of seizures was shortened in 10 patients and status epilepticus decreased in 6 [50].

The safety profile of stiripentol has been evaluated as good. Most adverse events are related to a significant increase in the plasma concentration of comedications, namely valproate, clobazam and Nor- clobazam after the addition of stiripentol; these effects disappear when the doses of comediction are adjusted. They include drowsiness, ataxia, nausea, abdominal pain, and loss of appetite resulting in weight loss [51].

In the long term, the frequency and the duration of seizures remain significantly reduced with a stiripentol-valproate-clobazam combination, as does the number of episodes of convulsive status epilepticus [52]. Efficacy seems to be better in younger patients, especially under the age of 2 years, with a strong benefit of stiripentol in shortening the prolonged convulsions. Given the presumed negative impact of early status epilepticus on cognitive outcome, 
stiripentol may therefore be beneficial as soon as the diagnosis of Dravet syndrome is confirmed [53].

\subsubsection{Topiramate}

Topiramate is a broad spectrum antiepileptic drug that is safe and effective in children [54] and is considered to be an alternative first line treatment in Dravet syndrome [55].

Efficacy for topiramate in patients with Dravet syndrome in observational studies is reported between 35 - 78\%. Side effects include word-finding difficulties, paraesthesia, weight loss/anorexia, kidney stones and rarely, narrow-angle glaucoma [56] [57].

In a retrospective study of 36 patients with Dravet syndrome that were considered to have poor control on stiripentol, $78 \%$ showed a more than $50 \%$ reduction in the frequency of generalized tonic-clonic seizures and status epilepticus after the introduction of topiramate, and $17 \%$ remained seizure-free for at least 4 months [58].

\subsubsection{Bromide}

Bromide was introduced by Doose in 1990 for the treatment of convulsive seizures in epilepsies in infancy. At the dose of $60-80 \mathrm{mg} / \mathrm{kg}, 32 \%$ became free of tonic-clonic seizures and another $47 \%$ experienced $>50 \%$ decrease in seizure frequency of those seizures [59]. The compound has no effect on minor seizures, that were even worsened in this original study. It has no effect on focal and tonic seizures. Acne, loss of appetite and weight, and fatigue are the main adverse effects described [60].

In a recent retrospective Japanese study of 276 patients diagnosed with Dravet syndrome, 160 (58\%) of which had a mutation in SCN1A gene, bromide was used in $41 \%$ of the SCN1Apositive patients and $21 \%$ of the SCN1A-negative patients. The responder rates for generalized clonic or tonic clonic seizures were $71 \%$ and $94 \%$ in SCN1A-positive and SCN1Anegative patients, respectively [61]. Because bromide monotherapy was not sufficient to control the multiple seizure types, it was typically used as an adjunct. In this study, the most 
common drug combinations were valproate-bromide, valproate-clonazepam-bromide, and valproate-clobazam-bromide. Bromide was rated to be the most effective and a well-tolerated drug, especially among SCN1A-negative patients.

Bromide was also - on par with clobazam - rated the most effective drug in patients with PCDH19 mutation. In a multicentre study of antiepileptic therapy in 58 female patients with PCDH19 mutation the most effective drugs after 3 months were also clobazam and bromide, with a responder rate of $68 \%$ and $67 \%$, respectively. PCDH19 mutations cause epilepsy and mental retardation limited to females (EFMR) as well as Dravet-like syndromes [62].

\subsubsection{Levetiracetam}

Levetiractam is widely used in paediatric epilepsy patients for treatment of generalised or focal seizures. There is evidence of efficacy for focal and generalised seizures in infancy [63]. It has got a favourable safety profile, and interactions with other antiepileptic drugs are not known. Reported responder rates in patient with Dravet syndrome however differ between 30\% [35] and $50 \%$ [64]. Children treated with levetiractam are at risk developing several behavioural side-effects such as aggression, hostility and nervousness. Side effects also include development of affective disorders like depression [65].

\subsubsection{Ketogenic diet}

The ketogenic diet is a high fat, restricted carbohydrate regime that has been used as a treatment for seizures since the 1920s, when it was designed to induce a similar metabolic response to fasting. After several modifications a growing number of studies reported similar success of classic or modified ketogenic diet protocols [66].

A randomised controlled trial to test the efficacy of the ketogenic diet was reported in 2008 . Data from 103 children were available for analysis 3 months after the start of treatment (54 on the ketogenic diet and 49 controls), the mean percentage of baseline seizures was significantly 
lower in the diet group than in the controls (62.0\% vs $136.9 \%, 75 \%$ decrease) [67]. The efficacy of the ketogenic diet for patients with Dravet syndrome is equally proven in at least two studies, which are summarised below.

In a retrospective study of 24 children with Dravet syndrome, two patients were seizure free, 10 had a $75-99 \%$ decrease in seizures, and 4 had a $50-74 \%$ decrease in seizures. Two years after starting the diet, 12 children had achieved $>75 \%$ decrease in their seizures. Eight of the 24 children (33.5\%) who initiated the diet discontinued it within the first year, five due lack of effectiveness, four because of severe vomiting. No complications were reported in the 16 patients who remained on the diet for $>2$ years [68]. A further study analysed retrospectively 32 children with genetically confirmed Dravet syndrome. The purpose of this study was to evaluate both effectiveness and tolerability in comparison with various antiepileptic drugs. Overall response to the ketogenic diet was $70 \%$ at 3 months and $60 \%$ at 12 months. No status epilepticus occurred while patients were on the diet, and the frequencies of prolonged generalized and myoclonic seizures were reduced. No severe side effects requiring withdrawal of the ketogenic diet were observed. Although the effect of the ketogenic diet was independent of age at initiation, it had to be withdrawn due to noncompliance more frequently in solid fed older children compared with infants treated with the liquid ketogenic formula [35]. The ketogenic diet was not significantly inferior to the current gold standard antiepileptic drug triple combination of stiripentol-valproate-clobazam (89\%), bromides (78\%), valproate alone (48\%), topiramate (35\%) and VNS (37\%) and significantly more effective than levetiracetam (30\%) [35].

Every form of ketogenic diet must be considered as an additional drug, it is not a natural treatment or diet and has well documented adverse effects and therefore requires close monitoring [69]. Side effects depend on the form of ketogenic diet and include hunger, vomiting, diarrhoea, lack of energy, constipation, and renal stones [70].

\subsection{Agents in development}




\subsubsection{Cannabidiol}

The two major neuroactive components in cannabis are the psychoactive D9tetrahydrocannabinol (D9-THC) and the nonpsychoactive cannabidiol. Unlike D9THC, cannabidiol does not activate CB1 and CB2 receptors, which likely accounts for its lack of psychotropic activity. Cannabidiol interacts with many other, non-endocannabinoid signalling systems: It is a "multitarget" drug [71]. It's exact antiseizure effect is unclear however. Preclinical evidence for anticonvulsant properties and a favourable side-effect profile support further development of cannabidiol-based treatments for epilepsy [72].

A prospective, open-label, expanded-access trial of cannabidiol at 11 independent epilepsy centres in the USA enrolled 214 patients; 162 (76\%) patients who had at least 12 weeks of follow-up after the first dose of cannabidiol were included in the safety and tolerability analysis, and $137(64 \%)$ patients were included in the efficacy analysis. $33(20 \%)$ patient in the safety group had Dravet syndrome. Cannabidiol was added to the baseline antiepileptic drug regimen, then up-titrated by $2-5 \mathrm{mg} / \mathrm{kg}$ once a week until intolerance or a maximum dose of $25 \mathrm{mg} / \mathrm{kg}$ per day was reached, at some sites to a maximum dose of $50 \mathrm{mg} / \mathrm{kg}$ per day.

For patients with Dravet syndrome in the efficacy group $(n=32)$, the median reduction in monthly motor seizures was $49.8 \% .16$ (50\%) patients had a reduction of $50 \%$ or greater and one $(3 \%)$ patient was free from motor seizures during the 3 months of treatment. A median reduction of $42.7 \%$ in monthly total seizures for all seizure types were recorded and, during the last 4 weeks of treatment, four (13\%) patients were free of motor seizures; these patients were also free of all other seizure types. There was a median change of $-69.2 \%$ in monthly tonic seizures $(n=6)$ and of $-46.7 \%$ in monthly tonic-clonic seizures $(n=29)$ [73].

$3 \%$ of the included patients discontinued the treatment because of side effects. Side effects reported in more than $5 \%$ of patients were somnolence, decreased appetite, diarrhoea, fatigue, convulsions, appetite changes, status epilepticus, lethargy, changes in concentrations of concomitant antiepileptic drugs, gait disturbance, and sedation. According to parent surveys, 
cannabidiol seemed to be tolerated well by patients and some reported added beneficial effects on sleep, alertness and mood [73].

These results are similar to previous smaller trials [74] [75].

Cannabidiol is a cytochrome P450 inhibitor. In comedication, serum levels of clobazam may increase by up to 60 to $80 \%$ [76]. $51 \%$ of the patients also taking clobazam reported somnolence or fatigue, whereas only 21 of the patients not taking clobazam reported somnolence. This alleviated with cannabidiol dose reduction which had no influence on seizure frequency [73]. The only predictor of response to cannabidiol in the open label study was comedication with clobazam. Both safety and efficacy need to be assessed through blinded, randomised controlled studies using consistent study medication of known composition. Such studies are now underway for cannabidiol use in patients with Dravet syndrome and Lennox Gastaut syndrome (Clinical Trials.gov, numbers NCT02224703, NCT02224560), and the results will be the start of any future research into use of cannabinoids in patients with epilepsy.

\subsubsection{Fenfluramine}

Fenfluramine (3-trifluoromethyl-N-ethylamphetamine), a derivate of amphetamine, causes the release of serotonin $(5-\mathrm{HT})$ by disrupting vesicular storage of the neurotransmitter and inhibiting its reuptake. It was recognized as an appetite suppressant in the mid-1960s and approved for this indication in the US in 1973 [77].

Ceulemans and colleagues retrospectively analysed the effect of fenfluramine in 12 patients with Dravet syndrome in 2010. Eleven of the patients had a mutation in SCN1A. Fenfluramine treatment was started at a mean age of 8 years (range: 1-16 years), administered at a mean of $0.34 \mathrm{mg} / \mathrm{kg} / \mathrm{day}$ (range: $0.12-0.90$ ), and added to each patient's current antiepilepsy regimen. The mean follow-up time after starting fenfluramine was 11 years and 4 months (range: 1-22 years). Seven of the 10 patients still taking the drug (70\%) had been seizure-free for at least 1 year prior to their most recent study visit, with a mean seizure-free period of 6.6 
years (range: $1-19)$. In addition, one patient (10\%) demonstrated a $75 \%$ reduction in seizure frequency and two others (20\%) had no significant effect on seizure frequency or severity. Cardiovascular follow-up (annually in the last 3 years of this analysis) showed no cases of pulmonary hypertension and no clinically significant thickening of the heart valves. Loss of appetite was reported in two patients [81].

Two new patients with Dravet syndrome, aged 10.8 and 1.2 years at study entry, have enrolled in the study. At the start of fenfluramine they were, respectively, taking VPA+TPM+CLB and VPA+CLB. After starting fenfluramine $(0.26$ and $0.77 \mathrm{mg} / \mathrm{kg} /$ day, respectively) they both demonstrated $a \geqslant 75 \%$ reduction in seizure frequency and disappearance of status epilepticus. Seizure-free periods were seen for 3 months in one child and 6 months in the other [82]. Both safety and efficacy need to be assessed through blinded, randomised controlled studies (Clinical Trials.gov, number NCT02682927). Zhang and colleagues recently demonstrated the anticonvulsive effect of fenfluramine in an antisense knockdown zebrafish model of Dravet syndrome. Incubation with fenfluramine significantly reduced the hyperactivity and the epileptiform discharges of the scn1Lab morphants [78].

\section{Conclusion}

Seizures of children with Dravet syndrome remain difficult to treat and require a combination of antiepileptic drugs in most cases. This aside, early appropriate treatment is the best way to decrease duration and frequency of seizures. Choice of appropriate antiepileptic drugs is important, however equally important is avoidance of medications that may aggravate seizures (eg lamotrigine, carbamazepine). Further, optimal and prompt treatment of fever, and prolonged seizures, with individualised treatment plans remain equally important. The debate continues as to the influence of ongoing seizures on neurodevelopmental progress, vs the underlying genetics of the disease. This aside, as the disease evolves with age, it is necessary to adapt treatment according to the change of seizure patterns and also side effects of medication as well as feasibility of use. Improvements may be seen even in later life with 
readjustment of medication. New and promising treatments remain on the horizon, but require further evaluation. Despite recent progress in pathophysiology and treatment, the prognosis of patients with Dravet syndrome with regard to both seizure control and neurocognitive outcome remains guarded. The potential influence of treatment in the longer term remains difficult to assess. 


\section{References}

[1] Dravet C, Bureau M, Oguni H et al. Severe myoclonic epilepsy in infancy (Dravet syndrome). In: Roger J, Bureau M, Dravet C, Genton P, Tassinari CA, Wolff P, editors. Epileptic syndromes in infancy, childhood and adolescence. 4th edn. France: John Libbey Eurotext; 2005. p. 89-113.

[2] Nabbout R, Chemaly N, Chipaux M,et al. Encephalopathy in children with Dravet syndrome is not a pure consequence of epilepsy. Orphanet J Rare Dis. 2013 Nov 13;8:176. doi: 10.1186/1750-1172-8-176

[3] Aras LM, Isla J, Mingorance-Le Meur A. The European patient with Dravet syndrome: results from a parent-reported survey on antiepileptic drug use in the European population with Dravet syndrome. Epilepsy Behav. 2015 Mar;44:104-9. doi: 0.1016/j.yebeh.2014.12.028 Epub 2015 Feb 6.

[4] Tro-Baumann B, von Spiczak S, Lotte J et al. A retrospective study of the relation between vaccination and occurrence of seizures in dravet syndrome. Epilepsia (2011) 52: 175-178. 10.1111/j.15281167.2010.02885.x; 10.1111/j.1528-1167.2010.02885.x.

[5] Verbeek NE, van der Maas NA, Jansen FE et al. Prevalence of SCN1A-related dravet syndrome among children reported with seizures following vaccination: a population-based ten-year cohort study. PLoS One. 2013 Jun 6;8(6):e65758. doi: 10.1371/journal.pone.0065758.

[6] McIntosh AM, McMahon J, Dibbens LM et al. Effects of vaccination on onset and outcome of Dravet syndrome: a retrospective study. Lancet Neurol. 2010 Jun;9(6):5928. doi: 10.1016/S1474-4422(10)70107-1. Epub 2010 May 4.

[7] Verbeek NE, van der Maas NA, Sonsma AC et al. Effect of vaccinations on seizure risk and disease course in Dravet syndrome. Neurology. 2015 Aug 18;85(7):596-603. doi: 10.1212/WNL.0000000000001855. Epub 2015 Jul 22.

[8] Le Gal F, Lebon S, Ramelli GP When is a child with status epilepticus likely to have Dravet syndrome? Epilepsy Res. 2014 May;108(4):740-7. doi: 10.1016/j.eplepsyres.2014.02.019 Epub 2014 Mar 12. 
[9] Dravet C, Daquin G, Battaglia D. Severe myoclonic epilepsy of infancy (Dravet syndrome). In Nikaronova M, Genton P, Sabers A (Eds) Topics in epilepsy. Long term evolution of epileptic encephalopathies. (2009) JohnLibbeyEurotext,Montrouge,pp.2938.

[10] Dravet C. The core dravet syndrome phenotype. Epilepsia 2011(52) Suppl 2: 39. 10.1111/j.1528-1167.2011.02994.x; 10.1111/j.1528-1167.2011.02994.x.

[11] Verbeek NE, Wassenaar M, van Campen JS et al. Seizure precipitants in Dravet syndrome: What events and activities are specifically provocative compared with other epilepsies? Epilepsy Behav. 2015 Jun;47:39-44. doi: 10.1016/j.yebeh.2015.05.008. Epub 2015 May 26.

[12] Wu YW, Sullivan J, McDaniel SS et al Incidence of Dravet Syndrome in a US Population. Pediatrics. 2015 Nov;136(5):e1310-5. doi: 10.1542/peds.2015-1807. Epub 2015 Oct 5.

[13] Brunklaus A, Ellis R, Reavey E et al. Prognostic, clinical and demographic features in SCN1A mutation-positive dravet syndrome. Brain (2012) 135: 2329-2336. $0.1093 /$ brain/aws151.

[14] Bayat A, Hjalgrim H, Møller RS. The incidence of SCN1A-related Dravet syndrome in Denmark is 1:22,000: A population-based study from 2004 to 2009 Epilepsia,56(4):e36-e39,2015 doi:10.1111/epi.12927

[15] Hirose S, Scheffer IE, Marini C, et al. Genetics Commission of the International League Against Epilepsy. SCN1A testing for epilepsy: application in clinical practice. Epilepsia. 2013 May;54(5):946-52. doi: 10.1111/epi.12168. Epub 2013 Apr 15.

[16] Oliva M, Berkovic SF, Petrou S. Sodium channels and the neurobiology of epilepsy Epilepsia,53(11):1849-1859,2012 doi:10.1111/j.1528-1167.2012.03631.x

[17] Depienne C, Trouillard O, Saint-Martin C et al. Spectrum of SCN1A gene mutations associated with Dravet syndrome: analysis of 333 patients. JMedGenet (2009) 46:183191. 
[18] Zuberi SM, Brunklaus A, Birch R et al Genotype-phenotype associations in SCN1A-related epilepsies. Neurology (2011) 76:594-600.

[19] Scheffer IE Diagnosis and long-term course of Dravet syndrome. Eur J Paediatr Neurol. 2012 Sep;16 Suppl 1:S5-8. doi: 10.1016/j.ejpn.2012.04.007. Epub 2012 Jun 16.

[20] Shi X, Yasumoto S, Nakagawa E, et al. Missense mutation of the sodium channel gene SCN2A causes Dravet syndrome. BrainDev (2009) 31:758-762.

[21] Battaglia D, Ricci D, Chieffo D, Guzzetta F. Outlining a core neuropsychological phenotype for Dravet syndrome. Epilepsy Res. 2016 Feb;120:91-7. doi:10.1016/j.eplepsyres.2015.11.020. Epub 2015 Dec 10. Review.

[22] Nabbout R, Chemaly N, Chipaux M, et al. Encephalopathy in children with Dravet syndrome is not a pure consequence of epilepsy. Orphanet J Rare Dis. 2013 Nov 13;8:176. doi: 10.1186/1750-1172-8-176.

[23] Wolff M, Cassé-Perrot C, Dravet C. Severe myoclonic epilepsy of infants (Dravet syndrome): natural history and neuropsychological findings. Epilepsia. 2006;47 Suppl 2:45-8.

[24] Ragona F, Brazzo D, De Giorgi I et al. Dravet syndrome: early clinical manifestations and cognitive outcome in 37 Italian patients. Brain Dev. 2010 Jan;32(1):71-7. doi: 10.1016/j.braindev.2009.09.014. Epub 2009 Oct 24.

[25] Catarino CB, Liu JY, Liagkouras I, et al. Dravet syndrome as epileptic encephalopathy: evidence from long-term course and neuropathology. Brain. 2011 Oct;134(Pt 10):2982-3010. doi: 10.1093/brain/awr129. Epub 2011 Jun 29

[26] Genton P, Velizarova R, Dravet C. Dravet syndrome: the long-term outcome. Epilepsia. 2011 Apr;52 Suppl 2:44-9. doi: 10.1111/j.1528-1167.2011.03001.x.

[27] Akiyama M, Kobayashi K, Yoshinaga H, Ohtsuka Y. A long-term follow-up study of Dravet Syndrome up to adulthood. Epilepsia 2010 51:1043-1052 
[28] Bureau M, Dalla Bernardina B. Electroencephalographic characteristics of Dravet syndrome. Epilepsia 2011 Apr;52 Suppl 2:13-23. doi: 10.1111/j.15281167.2011.02996.x

[29] Tsuda Y, Oguni H, Sakauchi M, Osawa M. An electroclinical study of absence seizures in Dravet syndrome. Epilepsy Res. 2013 Jan;103(1):88-96. doi: 10.1016/j.eplepsyres.2012.07.010. Epub 2012 Jul 21.

[30] Siegler Z, Barsi P, Neuwirth M et al. Hippocampal sclerosis in severe myoclonic epilepsy in infancy: a retrospective MRI study. Epilepsia 2005 (46):704-708

[31] Striano P, Mancardi MM, Biancheri R et al. Brain MRI findings in severe myoclonic epilepsy in infancy and genotype-phenotype correlations. Epilepsia (2007) 48:10921096

[32] Gaily E, Anttonen AK, Valanne L, et al Dravet syndrome: new potential genetic modifiers, imaging abnormalities, and ictal findings. Epilepsia. 2013 Sep;54(9):157785. doi: 10.1111/epi.12256. Epub 2013 Jun 28.

[33] Barba C, Parrini E, Coras R et al. Co-occurring malformations of cortical development and SCN1A gene mutations. Epilepsia 2014 Jul;55(7):1009-19. doi: 10.1111/epi.12658. Epub 2014 Jun 5.

[34] Perucca E. Pharmacological and therapeutic properties of valproate: a summary after 35 years of clinical experience. CNS Drugs. 2002;16(10):695-714. Review.

[35] Dressler A, Trimmel-Schwahofer P, Reithofer E et al. Efficacy and tolerability of the ketogenic diet in Dravet syndrome - Comparison with various standard antiepileptic drug regimen. Epilepsy Res. 2015 Jan;109:81-9. doi: 10.1016/j.eplepsyres.2014.10.014. Epub 2014 Oct 28.

[36] Shi XY, Tomonoh Y, Wang WZ et al. Epilepsy Genetic Study Group, Japan. Efficacy of antiepileptic drugs for the treatment of Dravet syndrome with different genotypes. Brain Dev. 2016 Jan;38(1):40-6. doi: 10.1016/j.braindev.2015.06.008. Epub 2015 Jul 13. 
[37] Aras LM, Isla J, Mingorance-Le Meur A. The European patient with Dravet syndrome: results from a parent-reported survey on antiepileptic drug use in the European population with Dravet syndrome. Epilepsy Behav. 2015 Mar;44:104-9. doi: 0.1016/j.yebeh.2014.12.028 Epub 2015 Feb 6.

[38] Tomson T, Battino D, Perucca E. Valproic acid after five decades of use in epilepsy: time to reconsider the indications of a time-honoured drug. Lancet Neurol. 2015 Dec 3. pii: S1474-4422(15)00314-2. doi: 10.1016/S1474-4422(15)00314-2. [Epub ahead of print] Review.

[39] Tomson T, Marson A, Boon P, Canevini MP et al. Valproate in the treatment of epilepsy in girls and women of childbearing potential. Epilepsia. 2015 Jul;56(7):100619. doi: 10.1111/epi.13021. Epub 2015 May 16. Review.

[40] Ng YT, Collins SD. Clobazam. Neurotherapeutics. 2007;4: 138-144

[41] Duchowny M, Cross H, Arzimanoglou A. Pediatric Epilepsy. 2012 McGraw-Hill Medical ISBN 13: 9780071496216

[42] Canadian Study Group for Childhood Epilepsy. Clobazam has equivalent efficacy to Carbamazepine and phenytoin as monotherapy for childhood epilepsy. Epilepsia. 1998 Sep;39(9):952-9.

[43] Perry MS, Bailey L, Malik S, et al. Clobazam for the treatment of intractable epilepsy in children. J Child Neurol. 2013 Jan;28(1):34-9. doi: 10.1177/0883073812461943. Epub 2012 Oct 30.

[44] Kalra V, Seth R, Mishra D, Saha NC. Clobazam in refractory childhood epilepsy. Indian J Pediatr. 2010;77:263-266

[45] Klehm J, Thome-Souza S, Sánchez Fernández I, et al. Clobazam: effect on frequency of seizures and safety profile in different subgroups of children with epilepsy. Pediatr Neurol. 2014 Jul;51(1):60-6. doi: 10.1016/j.pediatrneurol.2014.01.025. Epub 2014 Jan 16

[46] Singh A, Guberman AH, Boisvert D. Clobazam in long-term epilepsy treatment: sustained responders versus those developing tolerance. Epilepsia (1995) 36:798-803. 
[47] Chiron C, Marchand MC, Tran A, et al. Stiripentol in severe myoclonic epilepsy in infancy: a randomised placebo- controlled syndrome-dedicated trial. STICLO study group. Lancet 2000; 356: 1638-42.

[48] Giraud C, Treluyer JM, Rey E, et al. In vitro and in vivo inhibitory effect of stiripentol on clobazam metabolism. Drug Metab Dispos (2006) 34:608-611.

[49] Quilichini PP, Chiron C, Ben-Ari Y, Gozlan H. Stiripentol, a putative antiepileptic drug, enhances the duration of opening of GABA-A receptor channels. Epilepsia (2006) 47:704-716.

[50] Inoue Y, Ohtsuka Y, Oguni H., et al.. Stiripentol open study in Japanese patients with Dravet syndrome. Epilepsia (2009) 50, 2362-2368.

[51] Chiron C, Marchand MC, Tran A, et al. Stiripentol in severe myoclonic epilepsy in infancy: a randomised placebo- controlled syndrome-dedicated trial. STICLO study group. Lancet 2000; 356: 1638-42.

[52] Thanh TN, Chiron C, Dellatolas G, et al. Efficacite' et tolerance a` long terme du stiripentol dans le traitement de l'e'pilepsie myoclonique se've're du nourrisson (syndrome de Dravet). Arch Pediatr 2002; 9: 1120-7

[53] Chiron C. Current therapeutic procedures in Dravet syndrome.Dev Med Child Neurol. 2011 Apr;53 Suppl 2:16-8. doi: 10.1111/j.1469-8749.2011.03967.x. Review

[54] Wheless JW. Use of topiramate in childhood generalized seizure disorders.

J Child Neurol. 2000;15 Suppl 1:S7-13.

[55] McTague A, Cross JH. Treatment of epileptic encephalopathies. CNS Drugs. 2013 Mar;27(3):175-84. doi: 10.1007/s40263-013-0041-6. Review

[56] Nieto-Barrera M, Candau R, Nieto-Jimenez M, Correa A, del Portal LR. Topiramate in the treatment of severe myoclonic epilepsy in infancy. Seizure. 2000;9(8):590-4. doi:10.1053/seiz. 2000.0466.

[57] Coppola G, Capovilla G, Montagnini A et al. Topiramate as add-on drug in severe myoclonic epilepsy in infancy: an Italian multicenter open trial. Epilepsy Res. 2002;49(1):45-8. 
[58] Kröll-Seger J, Portilla P, Dulac O, Chiron C. Topiramate in the treatment of highly refractory patients with Dravet syndrome. Neuropediatrics. 2006 Dec;37(6):325-9.

[59] Ernst JP, Doose H, Baier WK. Bromides were effective in intractable epilepsy with generalized tonic-clonic seizures and onset in early childhood. BrainDev 1988 (10):385-388.

[60] Chiron C, Dulac O. The pharmacologic treatment of Dravet syndrome. Epilepsia (2011), 52 (Suppl.2):72-75, doi:10.1111/j.1528-1167.2011.03007.x

[61] Shi XY, Tomonoh Y, Wang WZ, et al. Epilepsy Genetic Study Group, Japan. Efficacy of antiepileptic drugs for the treatment of Dravet syndrome with different genotypes. Brain Dev. 2016 Jan;38(1):40-6. doi: 10.1016/j.braindev.2015.06.008. Epub 2015 Jul 13.

[62] Lotte J, Bast T, Borusiak P, Coppola A, Cross JH, et al. Effectiveness of antiepileptic therapy in patients with PCDH19 mutations. Seizure. 2016 Feb;35:106-10. doi: 10.1016/j.seizure.2016.01.006. Epub 2016 Jan 6

[63] Wilmshurst JM, Gaillard WD, Kollencheri PV et al. Summary of recommendations for the management of infantile seizures: Task Force Report for the ILAE Commission of Pediatrics Working Group: Epilepsia, 56(8):1185-1197, 2015 doi: 10.1111/epi.13057

[64] Striano P, Coppola A, Pezzella M et al. An open-label trial of levetiracetam in severe myoclonic epilepsy of infancy. Neurology. 2007;69(3):250-4. doi:10.1212/01.wnl.0000265222.24102.db.

[65] Halma E, de Louw AJ, Klinkenberg et al. Behavioral side-effects of levetiracetam in children with epilepsy: a systematic review. Seizure. 2014 Oct;23(9):685-91. doi: 10.1016/j.seizure.2014.06.004. Epub 2014 Jun 12. Review

[66] Neal EG, Cross JH. Efficacy of dietary treatments for epilepsy. J Hum Nutr Diet. 2010 Apr;23(2):113-9. doi: 10.1111/j.1365-277X.2010.01043.x. 
[67] Neal EG, Chaffe H, Schwartz RH et al. The ketogenic diet for the treatment of childhood epilepsy: a randomised controlled trial. Lancet Neurol. 2008 Jun;7(6):500-6. doi: 10.1016/S1474-4422(08)70092-9. Epub 2008 May 2.

[68] Caraballo RH. Nonpharmacologic treatments of Dravet syndrome: focus on the ketogenic diet. Epilepsia. 2011 Apr;52 Suppl 2:79-82. doi: 10.1111/j.1528167.2011.03009.x.

[69] McTague A, Cross JH. Treatment of epileptic encephalopathies.

CNS Drugs. 2013 Mar;27(3):175-84. doi: 10.1007/s40263-013-0041-6. Review

[70] Vezyroglou K, Cross JH. Targeted Treatment in Childhood Epilepsy Syndromes.

Curr Treat Options Neurol. 2016 Jun;18(6):29. doi: 10.1007/s11940-016-0407-4. Review

[71] Pertwee RG. The diverse CB1andCB2 receptor pharmacology of three plant cannabinoids: delta9-tetrahydrocannabinol, cannabidiol and delta9tetrahydrocannabivarin. BrJPharmacol 2008;153:199-215.

[72] Devinsky O, Cilio MR, Cross H, et al. Cannabidiol: pharmacology and potential therapeutic role in epilepsy and other neuropsychiatric disorders. Epilepsia. 2014 Jun;55(6):791-802. doi: 10.1111/epi.12631. Epub 2014 May 22. Review

[73] Devinsky O, Marsh E, Friedman D, et al. Cannabidiol in patients with treatmentresistant epilepsy: an open-label interventional trial. Lancet Neurol. 2016 Mar;15(3):270-8. doi: 10.1016/S1474-4422(15)00379-8. Epub 2015 Dec 24.

[74] Porter BE, Jacobson C. Report of a parent survey of cannabidiol enriched cannabis use in pediatric treatment-resistant epilepsy. Epilepsy Behav 2013; 29: 574-77.

[75] Gloss D, Vickrey B. Cannabinoids for epilepsy. Cochrane Database Syst Rev 2014; 3: CD009270.

[76] Geffrey AL, Pollack SF, Bruno PL, Thiele EA. Drug-drug interaction between clobazam and cannabidiol in children with refractory epilepsy. Epilepsia. 2015 Aug;56(8):1246-51. doi: 10.1111/epi.13060. Epub 2015 Jun 26. 
[77] Fuller R, Snoddy H and Robertson D. Mechanisms of effects of D-fenfluramine on brain serotonin metabolism in rats: uptake inhibition versus release. Pharmacol Biochem Behav (1988) 30: 715-721.

[78] Zhang Y, Kecskes A, Copmans D et al. Pharmacological characterization of an antisense knockdown zebrafish model of Dravet syndrome: inhibition of epileptic seizures by the serotonin agonist fenfluramine. PLoS One (2015) 10: e0125898

[79] Abenhaim L, Moride Y, Brenot F, Rich S et al. Appetite suppressant drugs and the risk of primary pulmonary hypertension. International Primary Pulmonary Hypertension Study Group. N Engl J Med (1996) 335: 609-616

[80] Connolly HM, Crary J, Mcgoon M et al. (1997) Valvular heart disease associated with fenfluramine-phentermine. N Engl J Med 337: 581-588

[81] Ceulemans B, Boel M, Leyssens K et al. Successful use of fenfluramine as an addon treatment for Dravet syndrome. Epilepsia (2012) 53: 1131-1139

[82] Schoonjans AS, Lagae L, Ceulemans B. Low-dose fenfluramine in the treatment of neurologic disorders: experience in Dravet syndrome.

Ther Adv Neurol Disord. 2015 Nov;8(6):328-38. doi: 10.1177/1756285615607726. 